\title{
Electrochromic Device with Three-state Optical Transformation Achieved by Modifying Electrode via Dip-coating Technique
}

\author{
Lu Wu ${ }^{\mathrm{a}}$, Dejiang Yang ${ }^{\mathrm{b}}$, Yiling Sun ${ }^{\mathrm{c}}$, Yong Xiang ${ }^{\star}, \mathrm{d}$ \\ School of Energy Science and Engineering, University of Electronic Science and Technology of \\ China, Chengdu 611731, China.
}

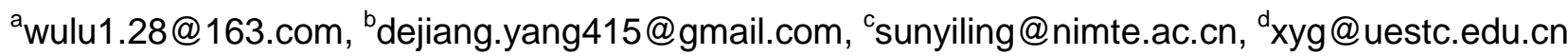

Keywords: Electrodeposition, Electrochromic Device, Dip-coating, Titanium Dioxide, Three States, Optical Transformation.

\begin{abstract}
A layer of $\mathrm{TiO}_{2}$ nanoparticles with size of $100 \mathrm{~nm}$ was deposited onto a fluorine-doped tin oxide (FTO) conducting electrode via dip-coating technique, followed by sintering treatment. Multi-state electrodeposition-based electrochromic device was subsequently fabricated by sandwiching gel electrolyte between a modified FTO electrode and a flat FTO electrode. When applied with a suitable voltage, the fabricated device changed its optical state from transparent to mirror or black depending on whether the silver nanoparticles were deposited on the unmodified or the modified FTO electrode. The silver was reversely dissolved into gel electrolyte once the applied voltage was removed. The fabricated device exhibited transmittance below $1 \%$ in the black state and reflectance over $70 \%$ in the mirror state. Moreover, the transmittance increased to $11 \%$ after 2000 cycles, indicative of good device stability.
\end{abstract}

\section{Introduction}

Electrochromic devices based on reversible electrodeposition have simple sandwich-type structure, with electrolyte and electrodeposition components interspersed between two transparent conducting electrodes. Their optical properties can be manipulated by depositing metal (copper, bismuth, plumbum, nickel, silver, etc.) onto transparent conducting electrodes in the presence of an applied voltage and, reversely, dissolving metal into electrolyte upon removal of the voltage [1-9]. Ag-based electrodeposition systems [9-12] have been widely investigated, but most of them only show black states through Ag deposition. Even though a mirror-like deposition via a silver dicyano ion complex or other additives has been achieved, the complexity and difficulty of achieving completely transparent state hinders its development and further application. Hence, it is necessary to develop Ag-based electrochromic devices with reversible multi-state using a simple and controllable method [13-15]. Araki et al. [13] deposited Ag onto an indium tin oxide (ITO) nanoparticle-modified ITO electrode via spin-coating and obtained reversible black/mirror state. Araki and his co-workers [14] achieved multi-color states for electrochromic device by controlling the growth of Ag grains under different voltages. In a previous study, our group fabricated an electrodeposition-based $\mathrm{Ag} / \mathrm{Cu}$ electrochromic device with a reversible three-state optical transformation by using conducting FTO electrode spin-coated with $\mathrm{TiO}_{2}$ nanoparticles [15]. Another technique to deposit thin film on the surface is dip-coating. Deepa, etc. [16] reported that compared with films fabricated via spin-coating, the $\mathrm{WO}_{3}$ thin film fabricated with dip-coating method showed improved optical performance. However, this deposition method has not yet been applied in the fabrication of electrodeposition-based $\mathrm{Ag} / \mathrm{Cu}$ electrochromic devices.

In this study, we fabricated an electrodeposition-based electrochromic device with reversible three-state optical transformation (among transparent, silver-mirror, and black) by depositing $\mathrm{TiO}_{2}$ nanoparticles onto FTO electrode using dip-coating technique. When applied with a suitable voltage, silver was deposited onto the flat FTO electrode or the $\mathrm{TiO}_{2}$ nanoparticles modified FTO electrode depending on the polarity of the applied voltage, resulting in change of optical states from transparent to mirror or black state. Reversely, Ag was dissolved into the gel electrolyte between two facing electrodes upon removal of the voltage. 


\section{Experimental}

To prepare the $\mathrm{TiO}_{2}$ nanoparticle dispersion, $\mathrm{TiO}_{2}$ nanoparticles (2.5 g) with lauric acid (0.25 g) and ethyl cellulose $(0.75 \mathrm{~g})$ were placed into a ball-mill jar firstly, mixed with terpineol $(16 \mathrm{~mL})$ and ethyl alcohol $(10 \mathrm{~mL})$ immediately before milling. $\mathrm{TiO}_{2}$ nanoparticle slurry was obtained after 50 min milling, followed by diluting the slurry with ethyl alcohol. To prepare the gel electrolyte, TBABr (806 mg), silver nitrate (85 mg), and copper chloride (13 mg) were dissolved in $10 \mathrm{~mL}$ of DMSO, followed by addition of PVB (1.32 g, $10 \mathrm{wt} \%) .10 \mathrm{ml}$ ethyl alcohol was added to $5 \mathrm{~mL}$ $\mathrm{TiO}_{2}$ nanoparticles dispersion and ultrasonically mixed for $30 \mathrm{~min}$. FTO electrode was fixed on the dip-coater, immersed into the above mentioned dispersion, and lifted with a speed of $1000 \mathrm{um} / \mathrm{s}$. Subsequently, the dip-coated FTO electrode was sintered for $30 \mathrm{~min}$ at $500^{\circ} \mathrm{C}$. In order to assemble an electrodeposition-based electrochromic device, Teflon sheets were used as spacers and were cut into $25 \times 25 \mathrm{~mm}$ with a $20 \times 20 \mathrm{~mm}$ hole in the middle. The gel electrolyte was contained in the hole of the Teflon sheet, a space formed when the Teflon sheet was sandwiched between a flat FTO electrode and a $\mathrm{TiO}_{2}$ nanoparticle-modified FTO electrode. Field-emission scanning electron microscope (FESEM, S-3400, Hitachi) was used to observe the morphology of the $\mathrm{TiO}_{2}$ thin film. Electrochemical test was conducted by using an electrochemical workstation (CHI660D, CHI) and the optical properties of the device were evaluated by measuring the transmittance and reflectance spectra using a UV-Vis spectrophotometer (Cary 5000, Agilent).

\section{Results and discussion}

As shown in Figure1, the fabricated electrodeposition-based electrochromic device exhibits three reversible optical states, including mirror, transparent, and black depending on the applied voltage. Without applied voltage, the device is transparent as shown in Figure 1a, in which the printed letters on the background can be clearly seen through the device. When a positive voltage $(+2.5 \mathrm{~V})$ is applied on the modified FTO working electrode, the device turns into mirror state as shown in Figure $1 b$, in which the reflection of the red pen can be clearly visible. The black state can be obtained by applying a negative voltage on the modified FTO electrode (Figure 1c).
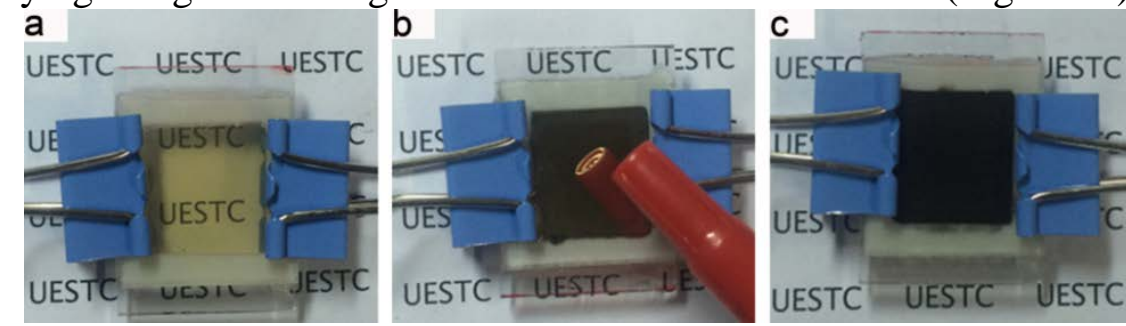

Figure 1. Photographs of the electrochromic device with transparent state (a), mirror state (b), and black state (c)

As illustrated in Figure 1, the deposition of an Ag layer on the flat or modified FTO electrode leads to absorption and multiple scattering of incidence light, resulting in mirror or black state. Therefore, the morphological feature of dip-coated $\mathrm{TiO}_{2}$ thin film is critical to the formation of the black state. Photograph and in-plane SEM image of the dip-coated $\mathrm{TiO}_{2}$ thin film are presented in Figure 2. FTO electrode turns from transparency to opaque after the deposition of $\mathrm{TiO}_{2}$ thin film, as illustrated in Figure 2a. Uniform pores and grains on the surface of the as-deposited $\mathrm{TiO}_{2}$ thin film can be observed (Figure 2b). Slight aggregation and agglomeration of $\mathrm{TiO}_{2}$ nanoparticles, formed during the dispersion of $\mathrm{TiO}_{2}$ nanoparticles in ethyl alcohol, the dip-coating of the $\mathrm{TiO}_{2}$ thin film, and the sintering treatment of as-prepared $\mathrm{TiO}_{2}$ thin film-can be observed.
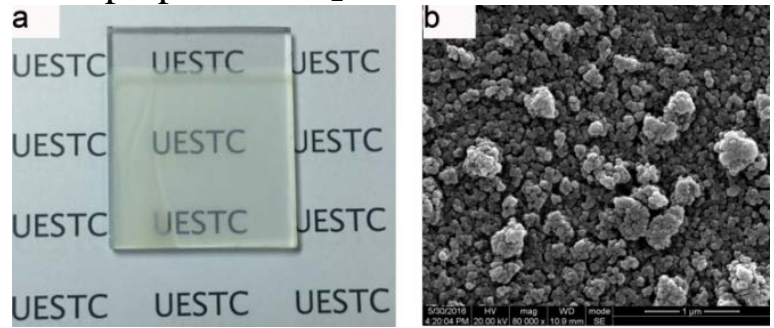

Figure 2. Photograph(a) and SEM image(b) of $\mathrm{TiO}_{2}$ nanoparticle-modified FTO electrodes via dip-coating 
Optical transmittance in the black state and reflectance in the mirror state were measured in the spectrum region from $400 \mathrm{~nm}$ to $800 \mathrm{~nm}$. For transmittance measurement, the negative pole and positive pole are connected to the flat FTO electrode and $\mathrm{TiO}_{2}$ nanoparticle-modified FTO electrode respectively, and a voltage of $-2.5 \mathrm{~V}$ was applied to the $\mathrm{TiO}_{2}$ nanoparticle-modified FTO electrode for 20 seconds to obtain the black state. For reflectance measurement, a voltage of $+2.5 \mathrm{~V}$ was applied to the $\mathrm{TiO}_{2}$ nanoparticle-modified FTO electrode for 90 seconds to obtain the mirror state. For the black state, transmittance of approximately $11 \%$ was measured (Figure 3a). It is worth mentioning that the obtained value does not correspond to the darkest state that can be achieved. For the mirror state, high reflectance of over $72 \%$ is observed (Figure $3 \mathrm{~b}$ ).
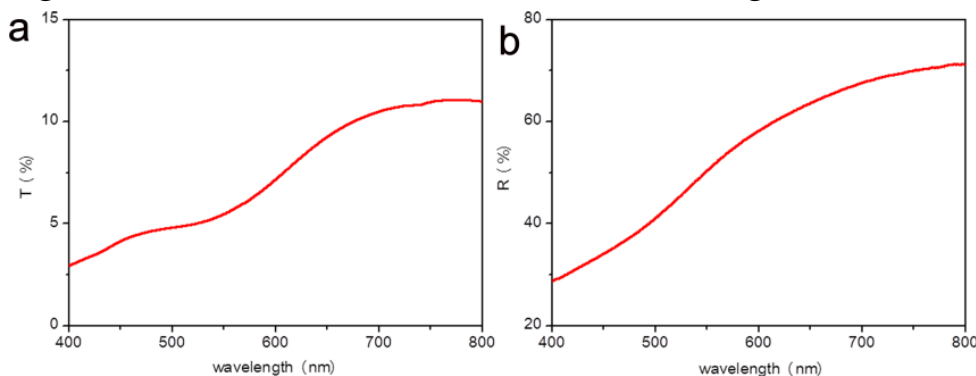

Figure3.Transmittance spectra in the black state (a), and reflectance spectra in the mirror state (b)

Usually, device failure occurs when an electrochromic device has been repeatedly switched between its colored and bleached states for a certain number of times because its life is limited by various faults and side reactions including transparent electrode failure, electrolyte depravation, decay of active layer etc. Herein, cycling stability was investigated by repeatedly applying sequential voltages to the electrodeposition-based electrochromic device. As shown in Figure 4, a voltage of $-2.5 \mathrm{~V}$ was applied to the $\mathrm{TiO}_{2}$ nanoparticle-modified device for 180 s to measure the transmittance variation at $700 \mathrm{~nm}$. Sequential voltages in the order of $-2.5 \mathrm{~V}(10 \mathrm{~s}), 0.5 \mathrm{~V}(30 \mathrm{~s}), 2.5$ $\mathrm{V}(10 \mathrm{~s})$ and $0.5 \mathrm{~V}(20 \mathrm{~s})$ were applied to the modified FTO electrode before the measurement. Each of the 500 cycles was taken as a measurement point to obtain the transmittance change with time. Upon application of the constant voltage onto the modified electrode for the first test cycle, the transmittance reduced sharply to a below $1 \%$ and remained fairly stable as a function of time. After 500, 1000, 1500 and 2000 cycles, the transmittance gradually increased with time, with more cycles leading to poorer stability. After 2000 cycles, the transmittance increases to $11 \%$ at $20 \mathrm{~min}$, indicative of good cycling stability.

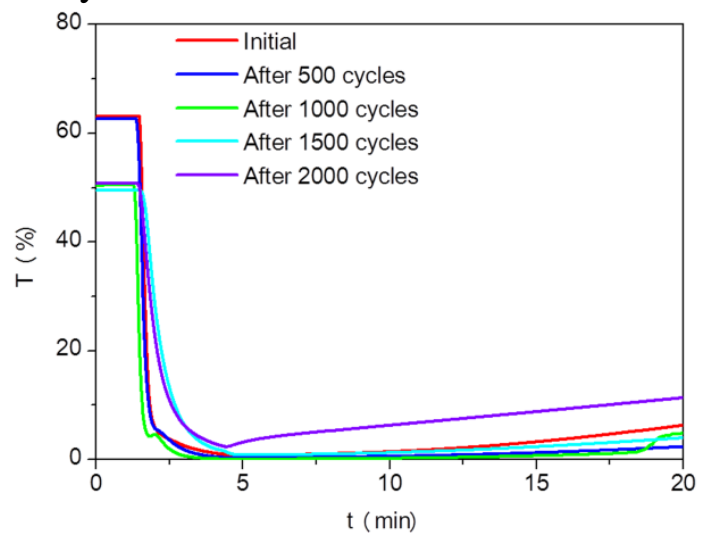

Figure 4. Transmittance variation of dip-coated devices at $700 \mathrm{~nm}$ after applying a sequence of voltages in the following order: -2.5 V (10s), $0.5 \mathrm{~V} \mathrm{(30} \mathrm{s),} 2.5 \mathrm{~V}(10 \mathrm{~s}), 0.5 \mathrm{~V}(20 \mathrm{~s})$

\section{Summary}

We have developed a facile and controllable dip-coating technique to deposit common and inexpensive $\mathrm{TiO}_{2}$ nanoparticle onto FTO conducting electrode. Rough $\mathrm{TiO}_{2}$ thin film with uniform pores and grains was obtained. The electrodeposition-based electrochromic device fabricated in the study achieved voltage-controlled and reversible three-state transformation, namely mirror, transparent and black states. Transmittance below $1 \%$ in the black state and reflectance over $70 \%$ in 
the mirror state for device was achieved. Moreover, satisfactory cycling stability after 2000 cycles was measured, which is better than that of spin-coated devices fabricated in our previous study. This $\mathrm{TiO}_{2}$ nanoparticle-modified device with reversible three-state optical transformation may find uses in various applications such as information displays and light-modulating devices.

\section{Acknowledgments}

This work was financially supported by the National Natural Science Foundation of China [grant number 51472044].

\section{References}

[1] J.P. Ziegler, B.M. Howard, Applications of reversible electrodeposition electrochromic devices, Sol. Energ. Mat. Sol. C. 39 (1995) 317-331.

[2] J.P. Ziegler, Status of reversible electrodeposition electrochromic devices, Sol. Energ. Mat. Sol. C.56 (1999) 477-493.

[3] D.A.A. Mello, M.R.S. Oliveira, L.C.S. Oliveira, S.C. Oliveira, Solid electrolytes for electrochromic devices based on reversible metal electrodeposition, Solar Energy Materials \& Solar Cells.103 (2012)17-24.

[4] C.O. Avellaneda, M.A. Napolitano, E.K. Kaibara, L.O.S. BulhÕes, Electrodeposition of lead on ITO electrode: influence of copper as an additive, Electrochim. Acta.50 (2005)1317-1321.

[5] S.I.C. Torresi, I.A. Carlos, Optical characterization of bismuth reversible electrodeposition, J. Electroanal. Chem. 414 (1996) 11-16.

[6] A. Imamura, M. Kimura, T. Kon, S. Sunohara, N. Kobayashi, Bi-based electrochromic cell with mediator for white/black imaging, Sol. Energ. Mat. Sol. C. 93 (2009) 2079-2082.

[7] M. Nakashima, T. Ebine, M. Shishikura, K. Hoshino, K. Kawai, K. Hatsusaka, Bismuth Electrochromic Device with High Paper-Like Quality and High Performances. Acs Appl. Mater. Interfaces. 2 (2010) 1471-1482.

[8] C.O. Avellaneda, M.A. Napolitano, E.K. Kaibara, L.O.S. BulhÕes, Electrodeposition of lead on ITO electrode: influence of copper as an additive, Electrochim. Acta. 50 (2005)1317-1321.

[9] M.R.S. Oliveira, D.A.A. Mello, E.A. Ponzio, S.C. Oliveira, KI effects on the reversible electrodeposition of silver on poly(ethylene oxide) for application in electrochromic devices, Electrochim. Acta.55 (2010) 3756-3765.

[10]T.Y. Kim, S.M. Cho, C.S. Ah, K.S. Suh, H. Ryu, H.Y. Chu, Electrochromic device for the reversible electrodeposition system, J. Soc. Inf. Display. 15 (2014)13-17.

[11]C. Park, S. Seo, H. Shin, B.D. Sarwade, J. Na, E. Kim, Switchable silver mirrors with long memory effects, Chem. Sci. 6 (2015) 596-602.

[12]I. Krastev, T. Valkova, A. Zielonka, Effect of electrolysis conditions on the deposition of silver-bismuth alloys, J. Appl Electrochem. 33 (2003) 1199-1204.

[13]S. Araki, K. Nakamura, K. Kobayashi, A. Tsuboi, N. Kobayashi, Electrochemical Optical-Modulation Device with Reversible Transformation between Transparent, Mirror, and Black, Adv. Mater. 24 (2012) OP122-OP126.

[14] A. Tsuboi, K. Nakamura, N. Kobayashi, A localized surface Plasmon resonance-based multicolor electrochromic device with electrochemically size-controlled silver nanoparticles, Adv. Mater. 25 (2013) 3197-3201. 
[15] T. Ye, Y. Xiang, H. Ji, C.J. Hu, G. Wu, Electrodeposition-based electrochromic devices with reversible three-state optical transformation by using titanium dioxide nanoparticle modified FTO electrode, RSC. Adv.6 (2016) 30769-30775.

[16]M. Deepa, T.K. Saxena, D.P. Singh, K.N. Sood, S.A. Agnihotry, Spin coated versus dip coated electrochromic tungsten oxide films: Structure, morphology, optical and electrochemical properties, Electrochim. Acta. 51 (2016)1974-1989. 